\title{
Joint Angles and Mutual Coupling Estimation Algorithm for Bistatic MIMO Radar
}

\author{
Jianfeng Li and Xiaofei Zhang \\ College of Electronic and Information Engineering, Nanjing University of Aeronautics and Astronautics, Nanjing 210016, China \\ Correspondence should be addressed to Jianfeng Li, lijianfengtin@126.com
}

Received 12 January 2012; Revised 18 April 2012; Accepted 25 April 2012

Academic Editor: Athanassios Manikas

Copyright () $2012 \mathrm{~J}$. Li and X. Zhang. This is an open access article distributed under the Creative Commons Attribution License, which permits unrestricted use, distribution, and reproduction in any medium, provided the original work is properly cited.

\begin{abstract}
We study the problem of angle estimation for a bistatic multiple-input multiple-output (MIMO) radar with unknown mutual coupling and proposed a joint algorithm for angles and mutual coupling estimation with the characteristics of uniform linear arrays and subspaces exploitation. We primarily obtain an initial estimate of DOA and DOD, then employ the local onedimensional searching to estimate exactly DOA and DOD, and finally evaluate the parameters of mutual coupling coefficients via the estimated angles. Exploiting twice of the one-dimensional local searching, our method has much lower computational cost than the algorithm in (Liu and Liao (2012)), and automatically obtains the paired two-dimensional angle estimation. Slightly better performance for angle estimation has been achieved via our scheme in contrast to (Liu and Liao (2012)), while the two methods indicate very close performance of mutual coupling estimation. The simulation results verify the algorithmic effectiveness of our scheme.
\end{abstract}

\section{Introduction}

Since multiple-input multiple-output (MIMO) radars exploit multiple antennas to manipulate parallel processing for transmitting diverse waveforms and receiving the reflected signals, several potential advantages over conventional phased-array radars such as angular diversity, flexible beampattern, and more degrees of freedom have been demonstrated in [1-4]. In contrast to other radar systems with a single transmit antenna, the additional degrees of freedom gained from MIMO radar has the capability of overcoming fading effect, enhancing spatial resolution, strengthening parameter identifiability, and improving target detection performance [5-8]. Several established angle estimation algorithms for MIMO radar comprise of the signal parameters estimation through the algorithms reliable on rotational invariance techniques (ESPRIT) [9], Capon [10], multiple signal classification (MUSIC) [11], parallel factor (PARAFAC) analysis [12], and adaptive PARAFAC [13], to name a few. However, most of the algorithms mentioned above take little concern for the problem of mutual coupling, which always exists in practical situations. The algorithm in the presence of unknown mutual coupling [14] for this practical situation achieves well estimation of angles and mutual coupling coefficients, while it is compulsory to perform twice of one-dimensional peak searching and additional pairing. Actually, the algorithmic complexity in [14] can be further reduced through the schemes of multidimensional data processing.

In our study, we present a low-complexity joint algorithm for the angles and mutual coupling coefficients estimation in bistatic MIMO radars. By employing the transformation of special matrix, we combine the signal subspace and noise subspace of the received data together to achieve the joint estimation of DOD and DOA. The angles can be automatically obtained via two local one-dimensional searching without the knowledge of mutual coupling, whose coefficients can be easily estimated after the angle estimation.

The remainder of this paper is structured as follows. Section 2 develops the data model for a MIMO radar system with unknown mutual coupling; Section 3 establishes the joint algorithm for joint angles and mutual coupling estimation in addition with the complexity analysis and CramérRao Bound (CRB). In Section 4, simulation results are presented to verify improvement for the proposed algorithm, while the final conclusions are summarized in Section 5. 
Notation. Bold lower (upper) case symbols denote column vectors or matrices; $(\cdot)^{T},(\cdot)^{*},(\cdot)^{H},(\cdot)^{-1}$, and $(\cdot)^{\dagger}$ denote the operators of transpose, conjugate, conjugate-transpose, inverse, and pseudoinverse, respectively, $\operatorname{diag}(\mathbf{v})$ stands for diagonal matrix whose diagonal element is a vector $\mathbf{v}$; $D_{n}(\cdot)$ denotes the operation of taking the $n$th row of the matrix to construct a diagonal matrix; $\mathbf{I}_{K}$ is a $K \times K$ identity matrix; $\otimes$ and $\odot$ represent the Kronecker product and Hadamard product, respectively; the real part of complex numbers is denoted by $\operatorname{Re}(\cdot)$ and the minimum elements of an array are denoted by $\min (\cdot) ; E[\cdot]$ stands for the expectation operator; $\operatorname{det}(\cdot)$ denotes the determinant of a matrix, $\operatorname{vec}(\cdot)$ denotes an operator stacking the columns of a matrix on top of each other.

\section{Data Model}

We consider a MIMO radar system equipped with $M$ transmit antennas and $N$ receive antennas, both of the antenna arrays are permuted as the uniform linear array (ULA), where the arrays of transmitter and receiver have been collocated in the $y$-axis with the interelement distance being half-wavelength [12]. The transmitted $M$-encoded pulse signals are orthogonal to each other; denote the $m$ th signal as $\mathbf{s}_{m}=\left[s_{m}(1), s_{m}(2), \ldots, s_{m}(L)\right]^{T}$, where $m=1,2, \ldots, M$, and $L$ is the length of the signal. We assume that there are $K$ uncorrelated targets, $\theta_{k}, \phi_{k}$ are direction of departure (DOD) and direction of arrival (DOA) of the $k$ th target with respect to the transmit array normal and the receive array normal, respectively. Assume the transmit and receive steering vectors are $\tilde{\mathbf{a}}_{t}\left(\theta_{k}\right)$ and $\widetilde{\mathbf{a}}_{r}\left(\phi_{k}\right)$, and hence, the signal arrive at the $k$ th target is written as $\widetilde{\mathbf{a}}_{t}^{T}\left(\theta_{k}\right) \mathbf{S}, k=1,2, \ldots, K$, where $\mathbf{S}=$ $\left[\mathbf{s}_{1}, \mathbf{s}_{2}, \ldots, \mathbf{s}_{M}\right]^{T} \in \mathbb{C}^{M \times L}$. Assume also that the reflection coefficients $\beta_{k}(t)$ and the phase shift $f_{k}$ are constant between pulse intervals, and the reflected signals of different targets are independent.

The receive data of the $N$ receive antennas of the $t$ th pulse can be denoted as

$$
\mathbf{Y}(t)=\sum_{k=1}^{K} \beta_{k}(t) e^{j 2 \pi f_{k} t} \widetilde{\mathbf{a}}_{r}\left(\phi_{k}\right) \tilde{\mathbf{a}}_{t}^{T}\left(\theta_{k}\right) \mathbf{S}+\mathbf{W}(t),
$$

where $\mathbf{Y}(t) \in \mathbb{C}^{N \times L}$ and $\mathbf{W}$ stand for the $N \times L$ receive data matrix and the noise matrix with identical determinants, respectively.

When each signal received by the $N$ antennas has been processed by a number of $M$ match filters [15], we obtain an $M \times N$ data matrix, which is equivalent to $\left[\mathbf{Y}(t) \mathbf{S}^{H}\right]^{T}$. After iterative vectorization for the input data, we finally construct the output of the $t$ th pulse as

$$
\mathbf{x}(t)=\operatorname{vec}\left(\left[\mathbf{Y}(t) \mathbf{S}^{H}\right]^{T}\right)
$$

Due to the orthogonality of the transmit signals towards each other, $\mathbf{S S}^{H}=\mathbf{I}$ is satisfied for the unitary matrix $\mathbf{S}$. Hence, we have

$$
\mathbf{x}(t)=\operatorname{vec}\left(\sum_{k=1}^{K} \beta_{k}(t) e^{j 2 \pi f_{k} t} \widetilde{\mathbf{a}}_{t}\left(\theta_{k}\right) \tilde{\mathbf{a}}_{r}^{T}\left(\phi_{k}\right)\right)+\operatorname{vec}\left(\left[\mathbf{W} \boldsymbol{S}^{H}\right]^{T}\right)
$$

$$
\begin{aligned}
& =\sum_{k=1}^{K} \beta_{k}(t) e^{j 2 \pi f_{k} t} \operatorname{vec}\left(\widetilde{\mathbf{a}}_{t}\left(\theta_{k}\right) \widetilde{\mathbf{a}}_{r}^{T}\left(\phi_{k}\right)\right)+\mathbf{n}(t) \\
& =\left[\widetilde{\mathbf{a}}_{r}\left(\phi_{1}\right) \otimes \widetilde{\mathbf{a}}_{t}\left(\theta_{1}\right), \ldots, \widetilde{\mathbf{a}}_{r}\left(\phi_{K}\right) \otimes \widetilde{\mathbf{a}}_{t}\left(\theta_{K}\right)\right] \mathbf{b}(t)+\mathbf{n}(t),
\end{aligned}
$$

where $\mathbf{b}(t)=\left[b_{1}(t), b_{2}(t), \ldots, b_{K}(t)\right]^{T} \in \mathbb{C}^{K \times 1}, b_{k}(t)=$ $\beta_{k}(t) e^{j 2 \pi f_{k} t} ; \mathbf{n}(t)$ is an $M N \times 1$ Gaussian white noise vector; $\widetilde{\mathbf{a}}_{r}\left(\phi_{k}\right)=\mathbf{C}_{r} \mathbf{a}_{r}\left(\phi_{k}\right) ; \tilde{\mathbf{a}}_{t}\left(\theta_{k}\right)=\mathbf{C}_{t} \mathbf{a}_{t}\left(\theta_{k}\right)$. It follows that $\mathbf{C}_{r} \in$ $\mathbb{C}^{N \times N}$ and $\mathbf{C}_{t} \in \mathbb{C}^{M \times M}$ represent the matrices containing the mutual coupling coefficients. According to the characteristics of ULA, if the elements have the same separation distance, the mutual coupling coefficients will be the same, so this will lead to two Toeplitz matrices as shown below.

$$
\begin{aligned}
\mathbf{C}_{r} & =\left[\begin{array}{ccccccccc}
c_{1}^{r} & \cdots & c_{n}^{r} & 0 & \cdots & & & & 0 \\
c_{2}^{r} & c_{1}^{r} & \cdots & c_{n}^{r} & 0 & \cdots & & & 0 \\
\vdots & & & & & & & & \\
c_{n}^{r} & \cdots & c_{1}^{r} & \cdots & c_{n}^{r} & 0 & \cdots & & 0 \\
0 & c_{n}^{r} & \cdots & c_{1}^{r} & \cdots & c_{n}^{r} & 0 & \cdots & 0 \\
\vdots & & & & & & & & \\
0 & \cdots & & & & 0 & c_{n}^{r} & \cdots & c_{1}^{r}
\end{array}\right], \\
\mathbf{C}_{t} & =\left[\begin{array}{ccccccccc}
c_{1}^{t} & \cdots & c_{m}^{t} & 0 & \cdots & & & & 0 \\
c_{2}^{t} & c_{1}^{t} & \cdots & c_{m}^{t} & 0 & \cdots & & & 0 \\
\vdots & & & & & & & & \\
c_{m}^{t} & \cdots & c_{1}^{t} & \cdots & c_{m}^{t} & 0 & \cdots & & 0 \\
0 & c_{m}^{t} & \cdots & c_{1}^{t} & \cdots & c_{m}^{t} & 0 & \cdots & 0 \\
\vdots & & & & & & & & \\
0 & \cdots & & & & 0 & c_{m}^{t} & \cdots & c_{1}^{t}
\end{array}\right],
\end{aligned}
$$

where $c_{p}^{r}(p=1 \cdots n)$ and $c_{q}^{t}(q=1 \cdots m)$ stand for the mutual coupling coefficients. $\mathbf{a}_{r}\left(\phi_{k}\right)$ and $\mathbf{a}_{t}\left(\theta_{k}\right)$ represent the receive and the transmit steering vectors for the $k$ th target, respectively. It is rewritten that

$$
\begin{aligned}
\mathbf{a}_{r}\left(\phi_{k}\right) & =\left[1, \exp \left(-j \pi \sin \phi_{k}\right), \ldots, \exp \left(-j(N-1) \pi \sin \phi_{k}\right)\right]^{T}, \\
\mathbf{a}_{t}\left(\theta_{k}\right) & =\left[1, \exp \left(-j \pi \sin \theta_{k}\right), \ldots, \exp \left(-j(M-1) \pi \sin \theta_{k}\right)\right]^{T} .
\end{aligned}
$$

Define $\tilde{\mathbf{A}}=\left[\widetilde{\mathbf{a}}_{r}\left(\phi_{1}\right) \otimes \widetilde{\mathbf{a}}_{t}\left(\theta_{1}\right), \ldots, \widetilde{\mathbf{a}}_{r}\left(\phi_{K}\right) \otimes \widetilde{\mathbf{a}}_{t}\left(\theta_{K}\right)\right]$, which can be denoted by

$$
\widetilde{\mathbf{A}}=\left[\begin{array}{c}
\tilde{\mathbf{A}}_{T} D_{1}\left(\widetilde{\mathbf{A}}_{R}\right) \\
\widetilde{\mathbf{A}}_{T} D_{2}\left(\widetilde{\mathbf{A}}_{R}\right) \\
\vdots \\
\widetilde{\mathbf{A}}_{T} D_{N}\left(\widetilde{\mathbf{A}}_{R}\right)
\end{array}\right],
$$

where $\tilde{\mathbf{A}}_{T}=\left[\tilde{\mathbf{a}}_{t}\left(\theta_{1}\right), \ldots, \tilde{\mathbf{a}}_{t}\left(\theta_{K}\right)\right] \in \mathbb{C}^{M \times K}, \tilde{\mathbf{A}}_{R}=$ $\left[\tilde{\mathbf{a}}_{r}\left(\phi_{1}\right), \ldots, \tilde{\mathbf{a}}_{r}\left(\phi_{K}\right)\right] \in \mathbb{C}^{N \times K}$. There exists a transformation matrix $\mathbf{H}$ corresponding to the finite number of row interchanged operations such that

$$
\widetilde{\mathbf{F}}=\mathbf{H} \tilde{\mathbf{A}}=\left[\widetilde{\mathbf{a}}_{t}\left(\theta_{1}\right) \otimes \widetilde{\mathbf{a}}_{r}\left(\phi_{1}\right), \ldots, \widetilde{\mathbf{a}}_{t}\left(\theta_{K}\right) \otimes \widetilde{\mathbf{a}}_{r}\left(\phi_{K}\right)\right] .
$$


And the matrix $\widetilde{\mathbf{F}}$ can be denoted by

$$
\widetilde{\mathbf{F}}=\left[\begin{array}{c}
\widetilde{\mathbf{A}}_{R} D_{1}\left(\widetilde{\mathbf{A}}_{T}\right) \\
\widetilde{\mathbf{A}}_{R} D_{2}\left(\widetilde{\mathbf{A}}_{T}\right) \\
\vdots \\
\widetilde{\mathbf{A}}_{R} D_{M}\left(\widetilde{\mathbf{A}}_{T}\right)
\end{array}\right] .
$$

By collecting $J$ samples and representing the received data as $\mathbf{X}=[\mathbf{x}(1), \mathbf{x}(2), \ldots, \mathbf{x}(J)]$, the transmitted signal matrix can now be generalized as

$$
\mathbf{X}=\tilde{\mathbf{A}} \mathbf{B}+\mathbf{N},
$$

where $\mathbf{N} \in \mathbb{C}^{M N \times J}$ stands for the noise matrix, $\mathbf{B}=$ $[\mathbf{b}(1), \mathbf{b}(2), \ldots, \mathbf{b}(J)] \in \mathbb{C}^{K \times J}$.

The covariance matrix of the received signal $\mathbf{R}=$ $E\left[\mathbf{x}(t) \mathbf{x}^{H}(t)\right]$ can be decomposed as

$$
\mathbf{R}=\mathbf{E}_{s} \mathbf{D}_{s} \mathbf{E}_{s}^{H}+\mathbf{E}_{n} \mathbf{D}_{n} \mathbf{E}_{n}^{H},
$$

where $\mathbf{D}_{s}$ denotes a $K \times K$ diagonal matrix formed by $K$ largest eigenvalues and $\mathbf{D}_{n}$ denotes a diagonal matrix formed by the rest $M N-K$ smaller eigenvalues. $\mathbf{E}_{s}$ and $\mathbf{E}_{n}$ represent the signal subspace and noise subspace, respectively, of which $\mathbf{E}_{s}$ stands for the eigenvectors corresponding to the $K$ largest eigenvalues $\mathbf{E}_{n}$ consists of the rest eigenvectors. In case of noise absence, the equation between $\mathbf{E}_{s}$ and $\widetilde{\mathbf{A}}$ can be formulated as

$$
\mathbf{E}_{s}=\tilde{\mathbf{A}} \mathbf{T}
$$

where $\mathbf{T}$ represents a nonsingular $K \times K$ matrix.

\section{Joint Angles and Mutual Coupling Estimation Algorithm}

3.1. Angles Estimation. According to the MUSIC method, the joint estimation of DOD and DOA can be achieved by the peak searching function as follows:

$$
\begin{aligned}
F\left(\theta, \phi, \mathbf{C}_{r}, \mathbf{C}_{t}\right) & =\frac{1}{\mathbf{Q}\left(\theta, \phi, \mathbf{C}_{r}, \mathbf{C}_{t}\right)} \\
& =\frac{1}{\left[\tilde{\mathbf{a}}_{r}(\phi) \otimes \tilde{\mathbf{a}}_{t}(\theta)\right]^{H} \mathbf{E}_{n} \mathbf{E}_{n}^{H}\left[\tilde{\mathbf{a}}_{r}(\phi) \otimes \tilde{\mathbf{a}}_{t}(\theta)\right]} .
\end{aligned}
$$

However, the method does not work if we cannot obtain $\mathbf{C}_{r}$ and $\mathbf{C}_{t}$. Even if the mutual coupling coefficients is already known, a two-dimensional searching is still required resulting into a considerably heavy computation burden.

With respect to the (4) and (9), the signal subspace matrix is represented by

$$
\mathbf{E}_{s}=\left[\begin{array}{c}
\widetilde{\mathbf{A}}_{T} D_{1}\left(\widetilde{\mathbf{A}}_{R}\right) \\
\widetilde{\mathbf{A}}_{T} D_{2}\left(\widetilde{\mathbf{A}}_{R}\right) \\
\vdots \\
\tilde{\mathbf{A}}_{T} D_{N}\left(\tilde{\mathbf{A}}_{R}\right)
\end{array}\right] \mathbf{T} .
$$

For which, it is partitioned as

$$
\mathbf{E}_{s}=\left[\begin{array}{c}
\mathbf{E}_{s 1} \\
\mathbf{E}_{s 2} \\
\vdots \\
\mathbf{E}_{s N}
\end{array}\right],
$$

where $\mathbf{E}_{s l} \in \mathbb{C}^{M \times K}, l=1, \ldots, N$. have

Taking the $n$th and $(n+1)$ th part into account, now we

$$
\begin{gathered}
\mathbf{E}_{s n}=\widetilde{\mathbf{A}}_{T} \operatorname{diag}\left(\mathbf{C}_{n}^{r} \mathbf{A}_{R}\right) \mathbf{T}, \\
\mathbf{E}_{s(n+1)}=\widetilde{\mathbf{A}}_{T} \operatorname{diag}\left(\mathbf{C}_{n+1}^{r} \mathbf{A}_{R}\right) \mathbf{T},
\end{gathered}
$$

where $\mathbf{C}_{n}^{r}$ is the $n$th row of $\mathbf{C}_{r}$. When $n$ satisfies $n \leq N / 2$, it is found that $\mathbf{C}_{n+1}^{r}$ is the right shift of $\mathbf{C}_{n}^{r}$, and hence,

$$
\operatorname{diag}\left(\mathbf{C}_{n+1}^{r} \mathbf{A}_{R}\right)=\operatorname{diag}\left(\mathbf{C}_{n}^{r} \mathbf{A}_{R}\right) \Phi_{r},
$$

where $\boldsymbol{\Phi}_{r}=\operatorname{diag}\left(\exp \left[-j \pi \sin \phi_{1}\right], \ldots, \exp \left[-j \pi \sin \phi_{K}\right]\right)$. Equation (12b) can be rewritten as

$$
\mathbf{E}_{s(n+1)}=\mathbf{E}_{s n} \mathbf{T}^{-1} \Phi_{r} \mathbf{T} .
$$

The diagonal elements of $\boldsymbol{\Phi}_{r}$ are the eigenvalues of $\mathbf{E}_{s n}^{\dagger} \mathbf{E}_{s(n+1)}$, and the corresponding eigenvectors are displayed as $\widehat{\mathbf{T}}^{-1}$. In case of noise absence, $\widehat{\mathbf{T}}^{-1}=\mathbf{T}^{-1} \boldsymbol{\Pi}$, where $\Pi$ is a column permutation matrix with $\Pi^{-1}=\Pi^{T}$. The estimation of $\boldsymbol{\Phi}_{r}$ is $\hat{\boldsymbol{\Phi}}_{r}=\boldsymbol{\Pi}^{T} \boldsymbol{\Phi}_{r} \boldsymbol{\Pi}$, and the DOA estimation can be obtained via

$$
\hat{\phi}_{k}^{i n i}=\sin ^{-1}\left(\frac{-\operatorname{angle}\left(\hat{u}_{k}\right)}{\pi}\right),
$$

where $\hat{u}_{k}$ is the $k$ th diagonal element of $\widehat{\boldsymbol{\Phi}}_{r}$.

The following matrix $\mathbf{E}_{c}$ stands for the column transformation of the direction matrix $\widetilde{\mathbf{F}}$ :

$$
\mathbf{E}_{c}=\mathrm{HE}_{s} \widehat{\mathbf{T}}^{-1}=\widetilde{\mathbf{F}} \Pi .
$$

This matrix $\mathbf{E}_{c}$ can now be similarly partitioned as

$$
\mathbf{E}_{c}=\left[\begin{array}{c}
\mathbf{E}_{c 1} \\
\mathbf{E}_{c 2} \\
\vdots \\
\mathbf{E}_{c M}
\end{array}\right],
$$

where $\mathbf{E}_{c l} \in \mathbb{C}^{N \times K}, l=1, \ldots, M$. Considering the $m$ th and $(m+1)$ th part of $\mathbf{E}_{c}$, by the similar method in equations from (9a) and (9b) to (13), the iterative matrix updates for $\mathbf{E}_{c m}$ now become

$$
\mathbf{E}_{c(m+1)}=\mathbf{E}_{c m} \boldsymbol{\Pi}^{T} \Phi_{t} \boldsymbol{\Pi},
$$

where $\boldsymbol{\Phi}_{t}=\operatorname{diag}\left(\exp \left[-j \pi \sin \theta_{1}\right], \ldots, \exp \left[-j \pi \sin \theta_{K}\right]\right)$. We assume that $\hat{v}_{k}$ is the $k$ th diagonal element of $\mathbf{E}_{c m}^{\dagger} \mathbf{E}_{c(m+1)}$, then the angle estimates can be achieved by

$$
\hat{\theta}_{k}^{i n i}=\sin ^{-1}\left(\frac{-\operatorname{angle}\left(\hat{v}_{k}\right)}{\pi}\right) .
$$

Therefore, the initial estimations of DOA and DOD have been obtained with the characteristics of automatic pairing. 
However, the estimation obtained by (15) and (16d) has low accuracy and bias, for it just employs partial information of the antennas, the more accurate estimation of the angles will be obtained below.

Referring to [14, Lemma 1], the estimation for the transmitted signals and their paired response can be obtained by

$$
\begin{aligned}
\tilde{\mathbf{a}}_{t}(\theta) & =\mathbf{C}_{t} \tilde{\mathbf{a}}_{t}(\theta)=\mathbf{T}_{t}(\theta) \mathbf{c}_{t}, \\
\widetilde{\mathbf{a}}_{r}(\phi) & =\mathbf{C}_{r} \widetilde{\mathbf{a}}_{r}(\phi)=\mathbf{T}_{r}(\phi) \mathbf{c}_{r},
\end{aligned}
$$

where $\mathbf{c}_{t}=\left[c_{1}^{t}, \ldots, c_{m}^{t}\right], \mathbf{c}_{r}=\left[c_{1}^{r}, \ldots, c_{n}^{r}\right], \mathbf{T}_{t}(\theta)=\mathbf{T}_{t 1}(\theta)+$ $\mathbf{T}_{t 2}(\theta)$, and the nonzero elements calculated from $\mathbf{T}_{t 1}(\theta) \in$ $\mathbb{C}^{M \times m}$ and $\mathbf{T}_{t 2}(\theta) \in \mathbb{C}^{M \times m}$ can now be written as

$$
\begin{gathered}
{\left[\mathbf{T}_{t 1}\right]_{i j}=\left(\mathbf{a}_{t}(\theta)\right)_{i+j-1}, \quad i+j \leq M+1,} \\
{\left[\mathbf{T}_{t 2}\right]_{i j}=\left(\mathbf{a}_{t}(\theta)\right)_{i-j+1}, \quad i \geq j \geq 2 .}
\end{gathered}
$$

It is shown for $\mathbf{Q}\left(\theta, \phi, \mathbf{C}_{r}, \mathbf{C}_{t}\right)$ that

$$
\mathbf{Q}\left(\theta, \phi, \mathbf{C}_{r}, \mathbf{C}_{t}\right)=\left(\mathbf{c}_{r} \otimes \mathbf{c}_{t}\right)^{H} \mathbf{Q}_{M}(\theta, \phi)\left(\mathbf{c}_{r} \otimes \mathbf{c}_{t}\right),
$$

where $\mathbf{Q}_{M}(\theta, \phi)=\left(\mathbf{T}_{r}(\phi) \otimes \mathbf{T}_{t}(\theta)\right)^{H} \mathbf{E}_{n} \mathbf{E}_{n}^{H}\left(\mathbf{T}_{r}(\phi) \otimes \mathbf{T}_{t}(\theta)\right)$.

Since $\mathbf{c}_{r} \otimes \mathbf{c}_{t} \neq \mathbf{0}$, and when $M N-K \geq m n$, we construct the function to search for the estimations of DOA and DOD [16] as follows:

$$
(\hat{\theta}, \hat{\phi})=\underset{\theta, \phi}{\arg \max } \frac{1}{\operatorname{det}\left(\mathbf{Q}_{M}(\theta, \phi)\right)} .
$$

Equation (20) requires a two-dimensional searching, which has a high complexity. Considering the initial estimation of DOD and DOA obtained in (15) and (16a), (16b), (16c), and (16d), we can use two one-dimensional searching to obtain more accurate estimation of DOD and DOA

$$
\hat{\theta}_{k}=\underset{\theta \in\left[\hat{\theta}_{k}^{\text {ini }}-\Delta \theta, \hat{\theta}_{k}^{\text {ini }}+\Delta \theta\right]}{\arg \operatorname{det}\left(\mathbf{Q}_{M}\left(\theta, \hat{\phi}_{k}^{\text {ini }}\right)\right)} .
$$

Throughout the local searching for $\theta$ within $\left[\hat{\theta}_{k}^{i n i}-\Delta \theta, \hat{\theta}_{k}^{i n i}+\right.$ $\Delta \theta$ ], where $\Delta \theta$ is a small value, we obtain more accurate estimation of the DOD $\hat{\theta}_{k}$. Similarly, $\phi_{k}$ can be also estimated from $\phi$ within $\left[\hat{\phi}_{k}^{i n i}-\Delta \phi, \hat{\phi}_{k}^{i n i}+\Delta \phi\right]$, where $\Delta \phi$ is a small value. Hence we have

$$
\hat{\phi}_{k}=\underset{\phi \in\left[\hat{\phi}_{k}^{n n i}-\Delta \phi, \hat{\phi}_{k}^{n i n}+\Delta \phi\right]}{\arg \max } \frac{1}{\operatorname{det}\left(\mathbf{Q}_{M}\left(\hat{\theta}_{k}, \phi\right)\right)},
$$

where $\hat{\theta}_{k}$ is estimation of $\theta_{k}$ via (21).

3.2. Mutual Coupling Estimation. According to (19), the estimates of $\mathbf{c}_{r t}=\mathbf{c}_{r} \otimes \mathbf{c}_{t}$ will be obtained from the minimum eigenvector of $(1 / K) \sum_{k=1}^{K} \mathbf{Q}_{M}\left(\hat{\theta}_{k}, \hat{\phi}_{k}\right)$. After normalizing $\mathbf{c}_{r t}\left(\mathbf{c}_{r t}(1)=1\right)$, and reshaping it to an $m \times n$ matrix $\mathbf{C}_{r t}$, we have

$$
\mathbf{C}_{r t}=\mathbf{c}_{t} \mathbf{c}_{r}^{T} .
$$

Then we obtain the estimation of $\mathbf{c}_{r}$ and $\mathbf{c}_{t}$ from the singular value decomposition of $\mathbf{C}_{r t}$. Let us assume $\mathbf{C}_{r t}=\mathbf{U S V}^{H}$ and

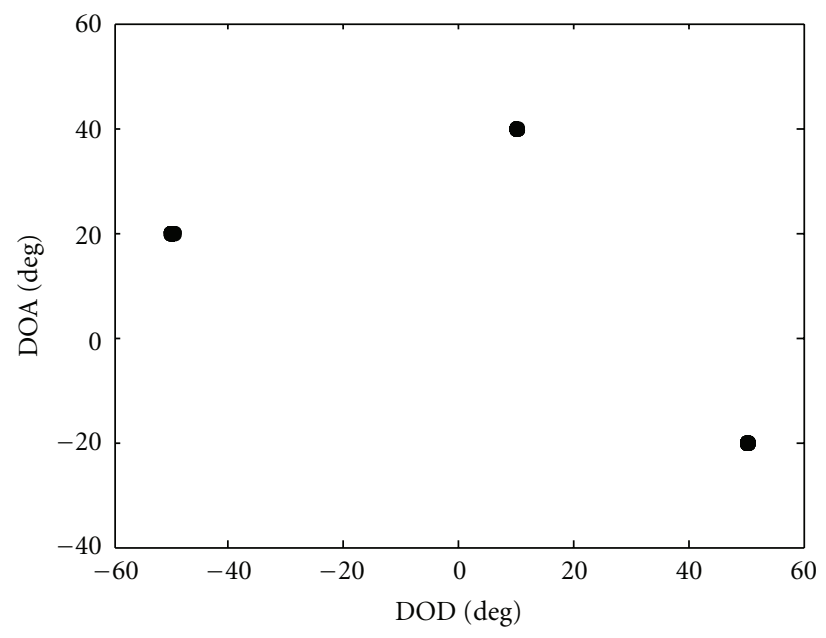

FIGURE 1: Angle estimation performance of the proposed algorithm with $\mathrm{SNR}=10 \mathrm{~dB}$.

the largest left singular vector is $\mathbf{u}_{t}$, while the largest right singular vector is $\mathbf{v}_{r}$, it is easy to construct that

$$
\begin{gathered}
\hat{\mathbf{c}}_{t}=\frac{\mathbf{u}_{t}}{\mathbf{u}_{t}(1)}, \\
\widehat{\mathbf{c}}_{r}=\left(\frac{\mathbf{v}_{r}}{\mathbf{v}_{r}(1)}\right)^{*} .
\end{gathered}
$$

Till now, we have achieved the proposal of our scheme for the joint angle and mutual coupling estimation in MIMO-radar. We show the major algorithmic steps as follows.

(1) Estimate the covariance matrix of the received data through $\widehat{\mathbf{R}}=\sum_{t=1}^{J} \mathbf{x}(t) \mathbf{x}^{H}(t)$.

(2) Perform eigenvalue decomposition of $\hat{\mathbf{R}}$ to get $\hat{\mathbf{E}}_{s}$ and $\hat{\mathbf{E}}_{N}$, and partition the matrix $\mathbf{E}_{s}$ to get $\mathbf{E}_{s 1}, \mathbf{E}_{s 2}, \ldots, \mathbf{E}_{s N}$. It follows to obtain $\hat{\phi}_{k}^{\text {ini }}$ from the eigenvalues of $\mathbf{E}_{s n}^{\dagger} \mathbf{E}_{s(n+1)}$ and $\hat{\mathbf{T}}$ from the corresponding eigenvectors.

(3) Compute $\mathbf{E}_{c}$, and get the initial estimation of the DOD $\hat{\theta}_{k}^{\text {ini }}$.

(4) Obtain the estimation of DOD $\hat{\theta}_{k}$ through onedimensional searching via (21) while keeping $\phi_{k}^{\text {ini }}$ fixed.

(5) Obtain the estimation of DOA $\hat{\phi}_{k}$ through onedimensional searching via (22) while keeping $\hat{\theta}_{k}$ fixed.

(6) Obtain the estimation of $\mathbf{C}_{r t}$ from the minimum eigenvector of $(1 / K) \sum_{k=1}^{K} \mathbf{Q}_{M}\left(\hat{\theta}_{k}, \hat{\phi}_{k}\right)$, and step further to get the estimation of $\mathbf{c}_{r}$ and $\mathbf{c}_{t}$ from the singular value decomposition of $\mathbf{C}_{r t}$ via (24).

3.3. Complexity Analysis and Cramer-Rao Bound (CRB). The proposed algorithm has lower complexity than the algorithm in [14]. Our scheme costs $O\left\{M^{2} N^{2} J+M^{3} N^{3}+2 K^{2} M+\right.$ $3 K^{3}+2 K^{2} N+K(2 N+2)+2 n_{1} K[M N m n+M N m n(M N-$ $\left.\left.K)+(m n) !+(M N-K) m^{2} n^{2}\right]+m^{3} n^{3}+\min \left(n^{2} m, m^{2} n\right)\right\}$, where $n_{1}$ is the number of steps within the local searching 


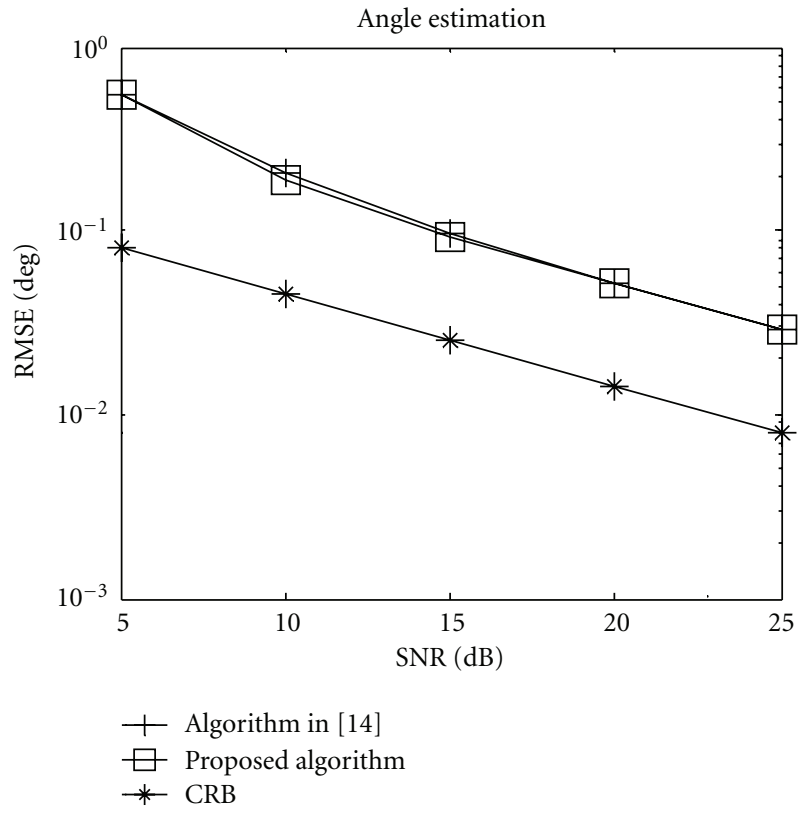

(a)

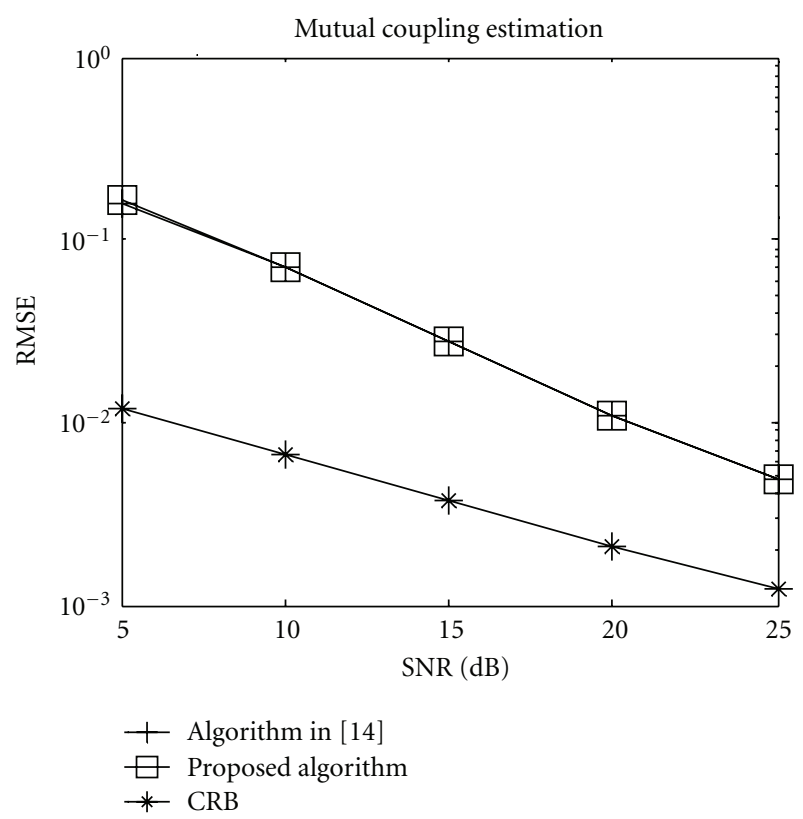

(b)

Figure 2: Angle and mutual coupling estimation performance comparison $(M=8, N=10, J=200, K=3)$.

range, while the algorithm in [14] requires $O\left\{M^{2} N^{2} J+\right.$ $M^{3} N^{3}+n_{2}\left[M^{2} N n(M N-K)+(M N-K) M^{2} n^{2}+2(M n) !+\right.$ $\left.N^{2} M m(M N-K)+N^{2} m^{2}(M N-K)\right]+K^{2}[M N m n+$ $\left.M N m n(M N-K)+(m n) !+(M N-K) m^{2} n^{2}\right]+m^{3} n^{3}+$ $\left.\min \left(n^{2} m, m^{2} n\right)\right\}$, where $n_{2}$ is the number of steps within the global searching range, and $n_{2} \gg n_{1}$.

According to [17], we derive the CRB for the joint angles estimation and mutual coupling algorithm in bistatic MIMO radar as follows:

$$
\mathrm{CRB}=\frac{\sigma^{2}}{2}\left\{\operatorname{Re}\left[\mathbf{D}^{H} \boldsymbol{\Pi}_{\mathbf{G}}^{\perp} \mathbf{D}\right]\right\}^{-1},
$$

where $\sigma^{2}$ stands for the covariance of the noise.

$$
\begin{aligned}
\mathbf{D}= & {\left[\frac{\partial \mathbf{u}}{\partial \theta_{1}}, \ldots, \frac{\partial \mathbf{u}}{\partial \theta_{K}}, \frac{\partial \mathbf{u}}{\partial \phi_{1}}, \ldots, \frac{\partial \mathbf{u}}{\partial \phi_{K}},\right.} \\
& \left.\frac{\partial \mathbf{u}}{\partial c_{2}^{t}}, \ldots, \frac{\partial \mathbf{u}}{\partial c_{m}^{t}}, \frac{\partial \mathbf{u}}{\partial c_{2}^{r}} \ldots, \frac{\partial \mathbf{u}}{\partial c_{n}^{r}}\right]
\end{aligned}
$$

with

$$
\mathbf{u}=\left[\begin{array}{c}
\tilde{\mathbf{A}} \mathbf{b}(1) \\
\vdots \\
\widetilde{\mathbf{A} b}(J)
\end{array}\right], \quad \boldsymbol{\Pi}_{\mathbf{G}}^{\perp}=\left[\begin{array}{ccc}
\Pi_{\tilde{\mathbf{A}}}^{\perp} & & 0 \\
& \ddots & \\
0 & & \boldsymbol{\Pi}_{\tilde{\mathbf{A}}}^{\perp}
\end{array}\right]
$$

with

$$
\Pi_{\widetilde{\mathbf{A}}}^{\perp}=\mathbf{I}_{M N}-\tilde{\mathbf{A}}\left(\widetilde{\mathbf{A}}^{H} \widetilde{\mathbf{A}}\right)^{-1} \widetilde{\mathbf{A}}^{H} .
$$

3.4. Advantages of the Proposed Algorithm. By compares and contrasts to [14], the algorithmic advantage of our approach can be briefly summarized as follows:

(1) Our approach can achieve the estimation for automatically paired two-dimensional angles, while the algorithm in [14] requires additional pairing.

(2) The proposed algorithm only requires the local onedimensional searching and hence has much lower complexity, while the algorithm in [14] urges two global one-dimensional searching.

(3) For the performance of angle and mutual coupling estimation, slightly improvement can be verified in our method than the one in [14].

(4) By fully exploiting both the signal subspace and the noise subspace, our method displays more validity and effectiveness, while the algorithm in [14] just employs the noise subspace.

\section{Simulation Results}

The Root mean square error (RMSE) of the angle and the RMSE of the mutual coupling coefficients [12] can be defined as

$$
\begin{aligned}
\operatorname{RMSE}_{a} & =\frac{1}{K} \sum_{k=1}^{K} \sqrt{\frac{1}{1000} \sum_{l=1}^{1000}\left[\left(\hat{\phi}_{k, l}-\phi_{k}\right)^{2}+\left(\hat{\theta}_{k, l}-\theta_{k}\right)^{2}\right]}, \\
\operatorname{RMSE}_{c} & =\sqrt{\frac{1}{1000} \sum_{l=1}^{1000}\left[\left\|\hat{\mathbf{c}}_{r, l}-\mathbf{c}_{r}\right\|_{F}^{2}+\left\|\hat{\mathbf{c}}_{t, l}-\mathbf{c}_{t}\right\|_{F}^{2}\right]},
\end{aligned}
$$

where $\hat{\theta}_{k, l}$ is the estimate of DOD, $\theta_{k}$ of the $l$ th Monte Carlo trial, $\hat{\phi}_{k, l}$ is the estimate of DOA $\phi_{k}$ of the $l$ th Monte Carlo trial, $\widehat{\mathbf{c}}_{r, l}$ is the estimate of $\mathbf{c}_{r}$ of the lth Monte Carlo trial, and $\hat{\mathbf{c}}_{t, l}$ is the estimate of $\mathbf{c}_{t}$ of the $l$ th Monte Carlo trial. We assume that there are $K=3$ targets with their angles correspondingly distributed as $\left(\theta_{1}, \phi_{1}\right)=\left(50^{\circ},-20^{\circ}\right),\left(\theta_{2}, \phi_{2}\right)=$ $\left(-50^{\circ}, 20^{\circ}\right),\left(\theta_{1}, \phi_{1}\right)=\left(10^{\circ}, 40^{\circ}\right) . \mathbf{c}_{t}=[1,0.1174+0.0577 j]$, and $\mathbf{c}_{r}=[1,-0.0121-0.1029 j]$. 


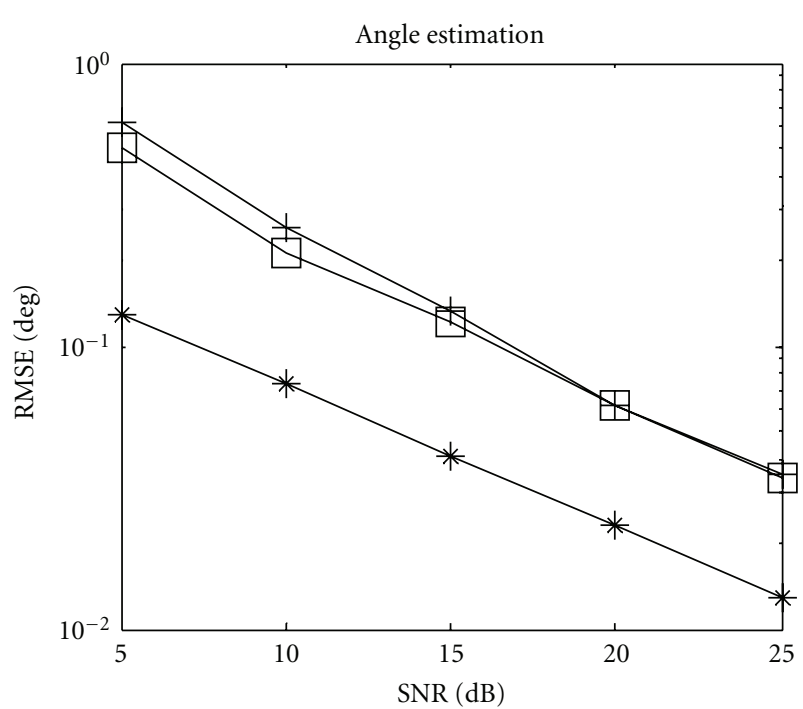

+ Algorithm in [14]
$\square$ Proposed algorithm
$*$ CRB

(a)

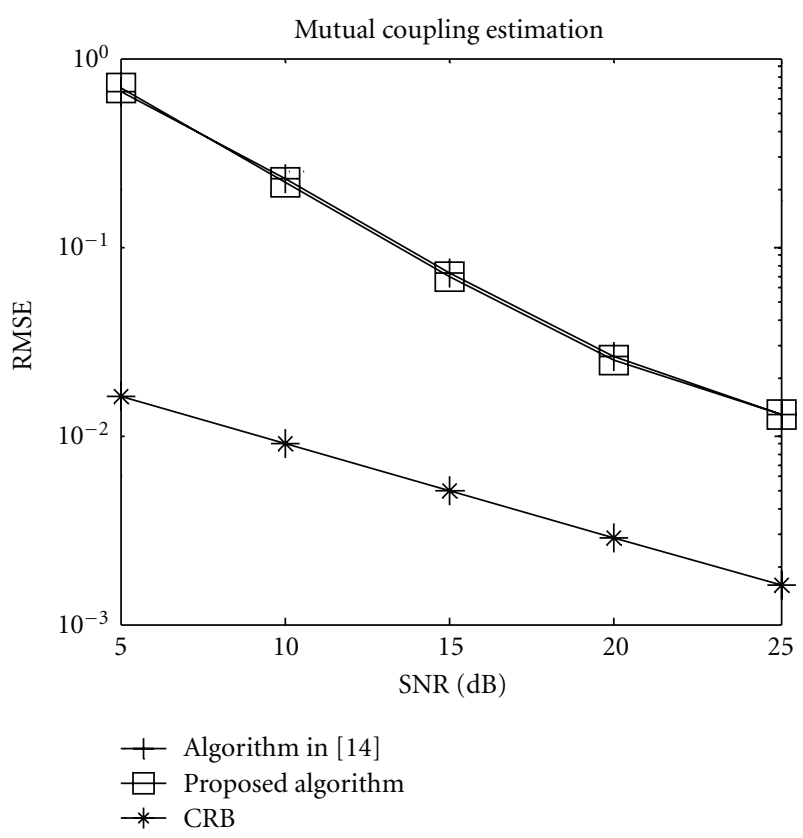

(b)

FIGURE 3: Angle and mutual coupling estimation performance comparison $(M=8, N=6, J=150, K=2)$.

Figure 1 depicts the estimation result of the proposed algorithm for all three targets with $M=8, N=10, J=200$, and $\mathrm{SNR}=10 \mathrm{~dB}$. It is explicit to show that the angles can be clearly observed.

We compare the proposed algorithm against the algorithm in [14] and CRB. Figures 2 and 3 present the comparison of the algorithms. From Figures 2 and 3, we can find that the angle estimation performance of the proposed algorithm is slightly better than the algorithm in [14], while our

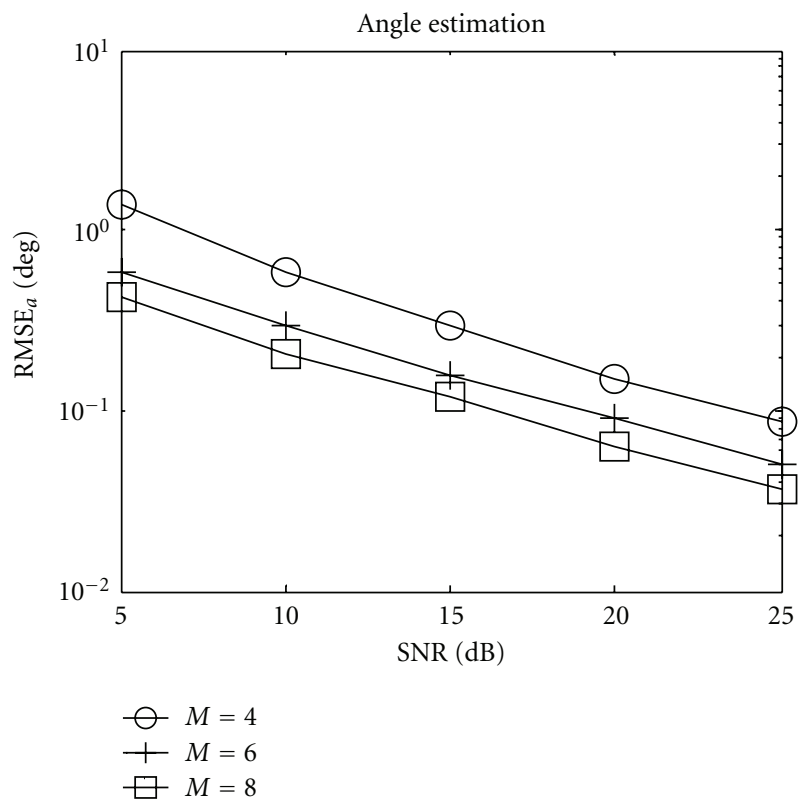

(a)

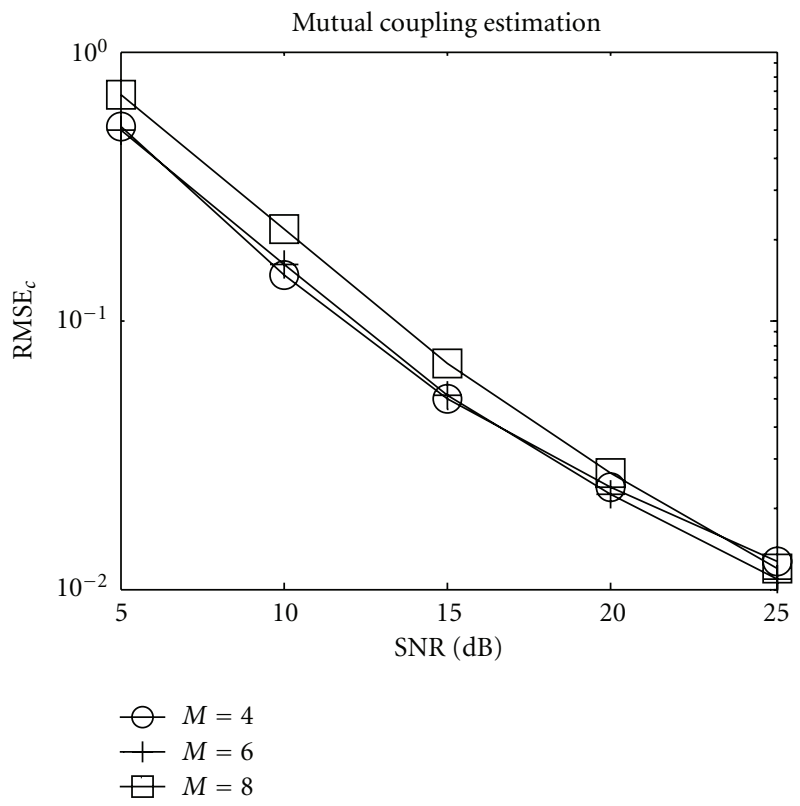

(b)

FIGURE 4: Angle estimation performance with $N=6$ and different values of $M$.

approach has very close performance for mutual coupling estimation to that of [14]. The reasons are (1) the method in [14] estimates the DOA and DOD separately, while the proposed considers the two angle's information simultaneously. (2) The proposed obtains an initial estimation firstly, which provides more information of the angles in estimation.

Figures 4 and 5 present the estimation performance of the proposed algorithm with $J=150, K=2$, and variable proportion of $M$ to $N$. From Figures 4 and 5, the angle estimation performance of the proposed algorithm is improved 


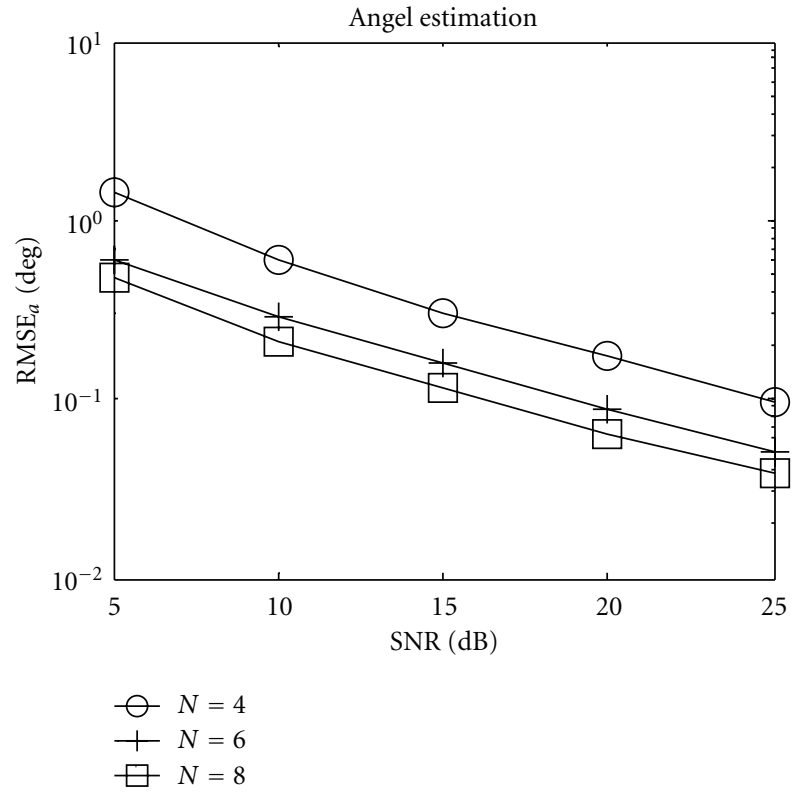

(a)

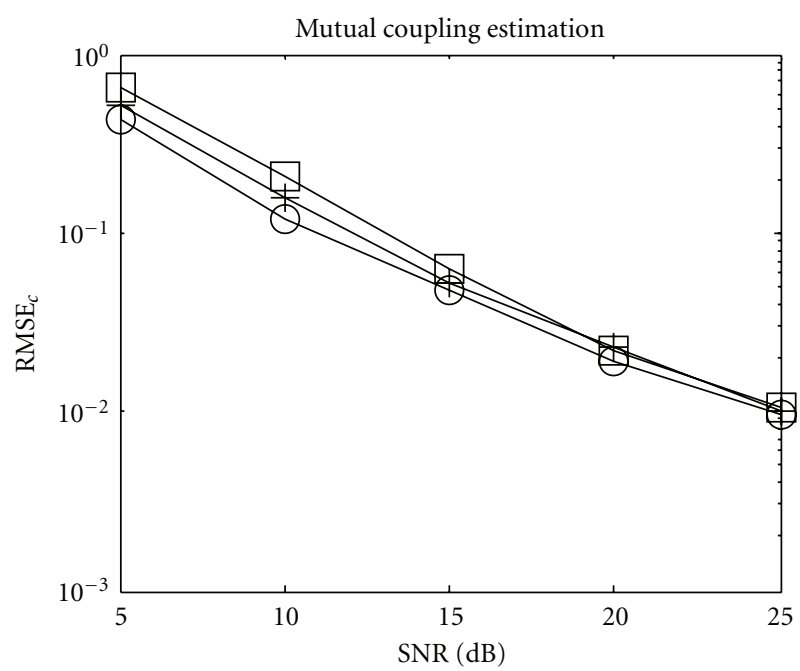

$\bigcirc N=4$

$+N=6$

$\square N=8$

(b)

Figure 5: Angle estimation performance with $M=8$ and different values of $N$.

with the increment of receive/transmit antenna numbers. Due to the diversity gain, multiple receive/transmit antennas improve angle estimation performance [12]. Meanwhile, it can be verified that the mutual coupling estimation performance of our method is improved with the decrement of receive/transmit antenna numbers. The dimensions of the mutual coupling matrix enlarge in condition that the number of receive/transmit antennas is increasing.

Figure 6 presents angle estimation performance of our scheme with $M=8, N=6, K=2$ and different values of $J$. It is indicated in Figure 6 that the angle estimation and

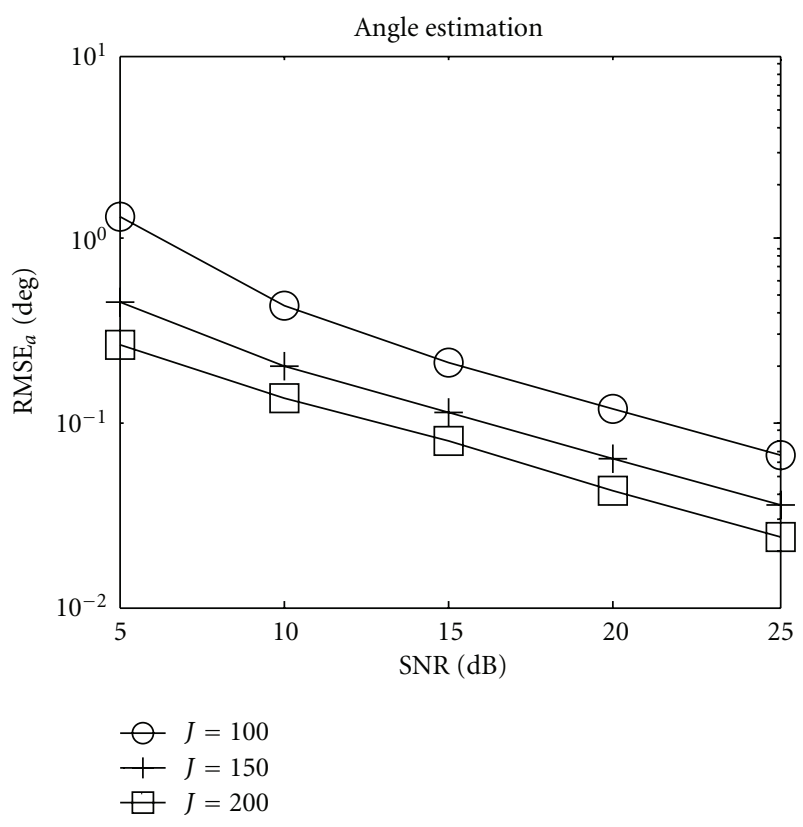

(a)

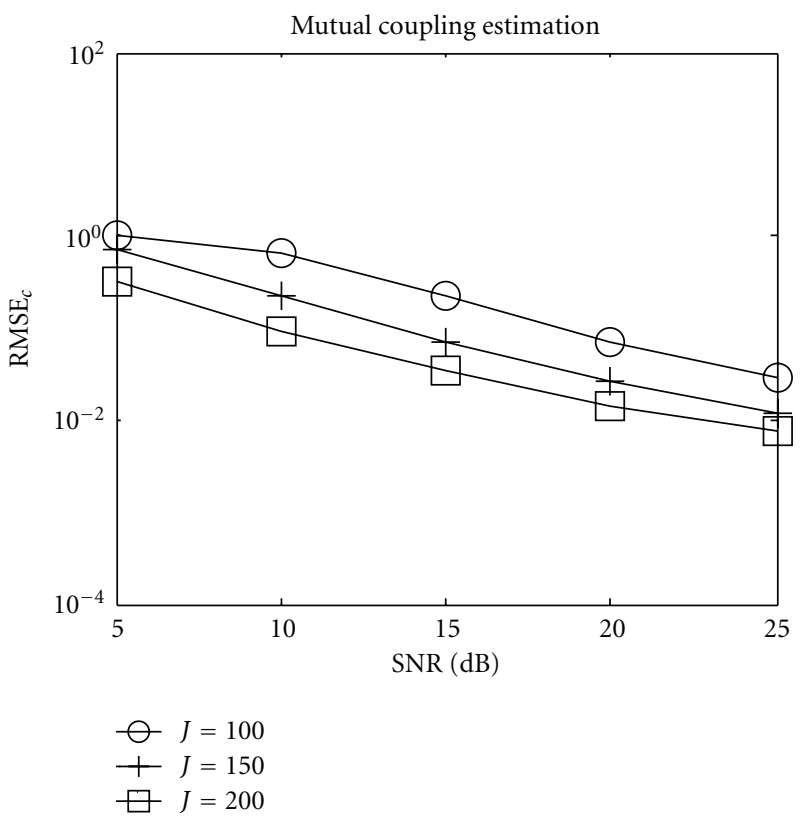

(b)

FIGURE 6: Angle estimation performance with different values of $J$.

mutual coupling estimation performance of the proposed algorithm is improved with $J$ increasing. When $J$ increases, we obtain a more accurate covariance matrix, and thereafter gain a more accurate subspace, which leads to more accurate angle estimation.

\section{Conclusion}

In this paper, we have presented a low-complexity algorithm for joint angles and mutual coupling estimation in bistatic MIMO radar with uniform linear arrays (ULAs). This 
method first obtains an initial estimate of DOA and DOD, then employs the local one-dimensional searching to achieve exact estimates for DOA and DOD, and finally estimates mutual coupling coefficients via the estimated angles. Automatically paired two-dimensional angle estimation can be also performed by our proposed algorithm. We have reduced the algorithmic complexity in comparison with that of [14] and established better performance of angle estimation.

\section{Acknowledgments}

This work is supported by China NSF Grants (60801052) and the Fundamental Research Funds for the Central Universities (NZ2012010, kfjj120115, kfjj20110215).

\section{References}

[1] E. Fishler, A. Haimovich, R. Blum, D. Chizhik, L. Cimini, and R. Valenzuela, "MIMO radar: an idea whose time has come," in Proceedings of the IEEE Radar Conference, pp. 71-78, April 2004.

[2] J. Li and P. Stoica, "MIMO radar-diversity means superiority," in Proceedings of the 14th Adaptive Sensor Array Processing Workshop (ASAP '06), pp. 1-6, Lincoln Lab, Mass, USA, 2006.

[3] X.-R. Li, Z. Zhang, W.-X. Mao, X.-M. Wang, J. Lu, and W.-S. Wang, "A study of frequency diversity MIMO radar beamforming," in Proceedings of the IEEE 10th International Conference on Signal Processing (ICSP '10), pp. 352-356, 2010.

[4] R. Sharma, "Analysis of MIMO radar ambiguity functions and implications on clear region," in Proceedings of the IEEE International Radar Conference (RADAR '10), pp. 544-548, May 2010.

[5] J. Li, G. Liao, and H. Griffiths, "Bistatic MIMO radar spacetime adaptive processing," in Proceedings of the IEEE Radar Conference (RadarCon '11), pp. 498-501, Westin Crown Center in Kansas City, 2011.

[6] X. H. Wu, A. A. Kishk, and A. W. Glisson, "MIMOOFDM radar for direction estimation," IET Radar, Sonar \& Navigation, vol. 4, no. 1, pp. 28-36, 2010.

[7] J. Li, P. Stoica, L. Xu, and W. Roberts, "On parameter identifiability of MIMO radar," IEEE Signal Processing Letters, vol. 14, no. 12, pp. 968-971, 2007.

[8] J. Li, G. Liao, K. Ma, and C. Zeng, "Waveform decorrelation for multitarget localization in bistatic MIMO radar systems," in Proceedings of the IEEE International Radar Conference (RADAR '10), pp. 21-24, May 2010.

[9] J. Chen, H. Gu, and W. Su, "Angle estimation using ESPRIT without pairing in MIMO radar," Electronics Letters, vol. 44, no. 24, pp. 1422-1423, 2008.

[10] H. Yan, J. Li, and G. Liao, "Multitarget identification and localization using bistatic MIMO radar systems" EURASIP Journal on Advances in Signal Processing, vol. 2008, Article ID 283483, 8 pages, 2008.

[11] X. Gao, X. Zhang, G. Feng, Z. Wang, and D. Xu, "On the MUSIC-derived approaches of angle estimation for bistatic MIMO radar," in Proceedings of the International Conference on Wireless Networks and Information Systems (WNIS '09), pp. 343-346, Shanghai, China, December 2009.

[12] X. Zhang, Z. Xu, L. Xu, and D. Xu, "Trilinear decompositionbased transmit angle and receive angle estimation for multiple-input multiple-output radar," IET Radar, Sonar \& Navigation, vol. 5, no. 6, pp. 626-631, 2011.
[13] D. Nion and N. D. Sidiropoulos, "Adaptive algorithms to track the PARAFAC decomposition of a third-order tensor," IEEE Transactions on Signal Processing, vol. 57, no. 6, pp. 2299-2310, 2009.

[14] X. Liu and G. Liao, "Direction finding and mutual coupling estimation for bistatic MIMO radar," Signal Processing, vol. 92, no. 2, pp. 517-522, 2012.

[15] I. Bekkerman and J. Tabrikian, "Target detection and localization using MIMO radars and sonars," IEEE Transactions on Signal Processing, vol. 54, no. 10, pp. 3873-3883, 2006.

[16] B. H. Wang, Y. L. Wang, and H. Chen, "A robust DOA estimation algorithm for uniform linear array in the presence of mutual coupling," in Proceedings of the IEEE International Antennas and Propagation Symposium and USNC/CNC/URSI North American Radio Science Meeting, pp. 924-927, Columbus, Ohio, USA, June 2003.

[17] P. Stoica and A. Nehorai, "Performance study of conditional and unconditional direction-of-arrival estimation," IEEE Transactions on Acoustics, Speech, and Signal Processing, vol. 38, no. 10, pp. 1783-1795, 1990. 

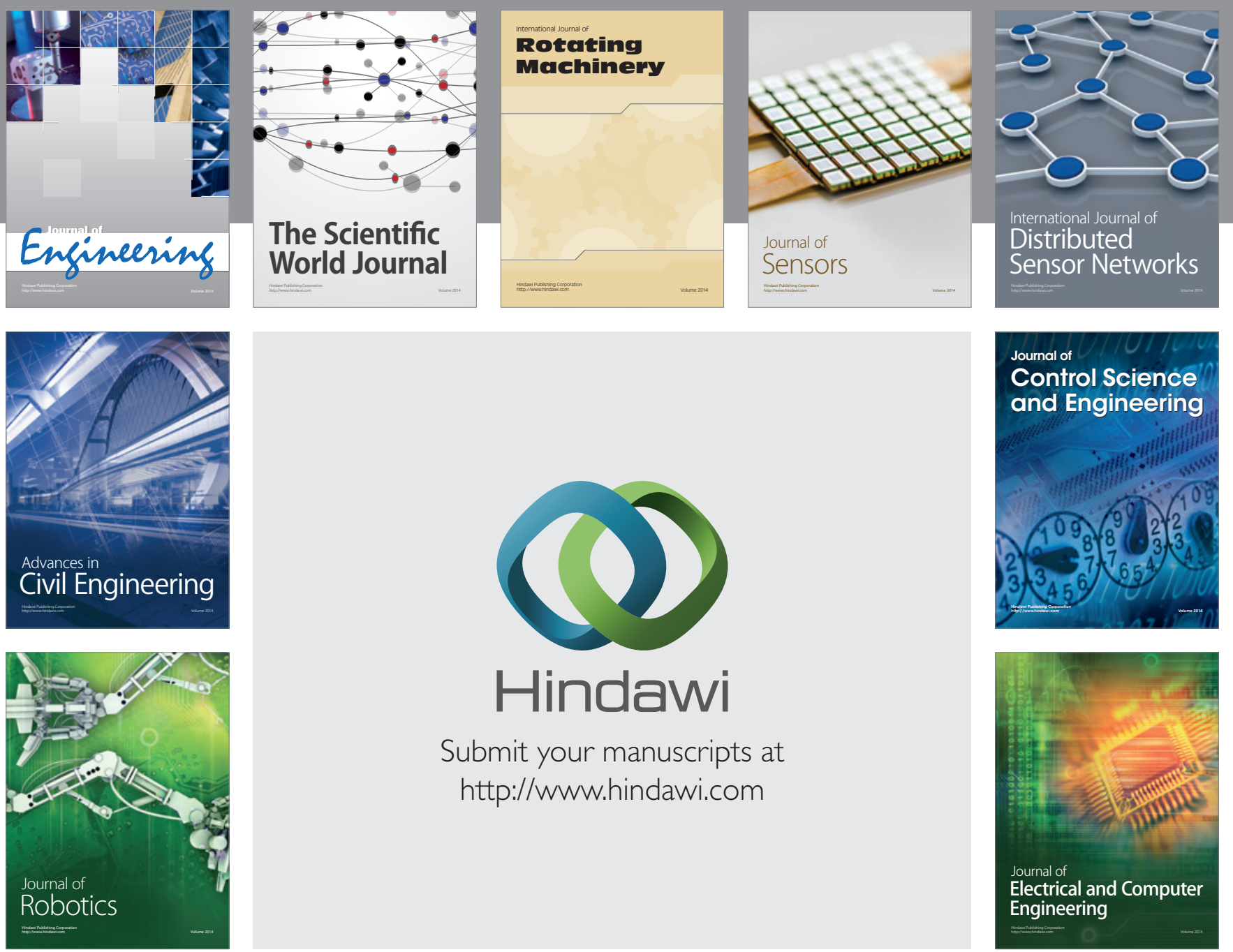

Submit your manuscripts at

http://www.hindawi.com
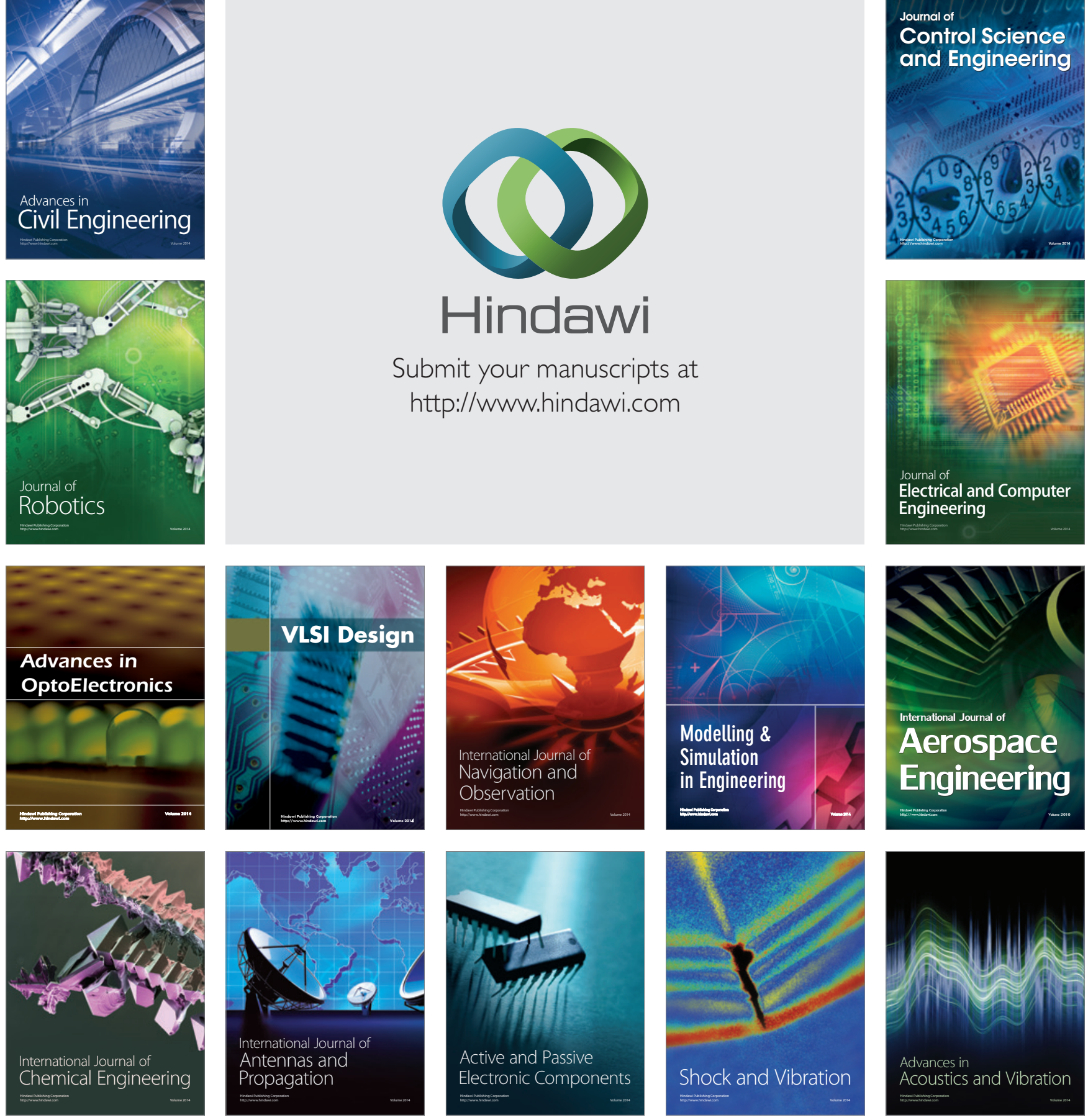